\title{
On the persistence of memory: do initial conditions impact vortex formation?
}

\author{
Jochen Kriegseis ${ }^{1}$, Matthias Kinzel ${ }^{2}$ and David E. Rival ${ }^{1, \dagger}$ \\ ${ }^{1}$ Department of Mechanical Engineering, University of Calgary, Calgary, T2N 1N4, Canada \\ ${ }^{2}$ Graduate Aeronautical Laboratories, California Institute of Technology, Pasadena, CA 91125, USA
}

(Received 24 March 2013; revised 19 August 2013; accepted 27 September 2013; first published online 1 November 2013)

An investigation into redistribution of vorticity for rapidly accelerating plates with varying kinematics and initial conditions has been performed. Both threedimensional particle tracking velocimetry and direct force measurements were applied simultaneously. The effective velocity of the feeding shear layer has been identified as the appropriate characteristic velocity rather than the commonly used plunge or free stream velocity. Based on this new normalization for circulation, it has been demonstrated that the existence of initial boundary-layer vorticity on the plunging plate - at least in the near-midplane region - does not contribute to the eventual vortex formation process. In accordance with the literature, however, the tip vortex positioning relative to the plate surface has been identified as an important contributor in the overall force production, particularly once the plate acceleration has ceased.

Key words: low-Reynolds-number flows, vortex flows

\section{Introduction}

In the treatise of Morton (1984) on the generation of vorticity, it is argued that, for a thin flat plate in uniform flow, vorticity is produced only at the leading edge and thus the boundary layer is formed due to diffusion and advection alone. In contrast, for Stokes' first problem, in which a thin flat plate is impulsively started from rest in the streamwise direction, it is conjectured that vorticity is generated across the plate surface instantaneously (see e.g. White 1991). Thereafter, vorticity is diffused off the surface but the total circulation remains constant. However, for non-slender shapes and/or rapid lateral motion of a body, a strong pressure gradient exists. Here, vorticity is presumably generated via tangential pressure gradients along the surface of the body, as proposed by Morton (1984). Unfortunately, little is understood regarding the subsequent two-dimensional redistribution and/or three-dimensional reorientation of this vorticity layer. One unique study performed by Gendrich (1999) on a pitching NACA0012 profile showed that the near-surface fluid - and thus vorticity - interacted with the leading-edge vortex growth during the dynamic stall process. Another example of boundary-layer vorticity reorientation was measured by Wibawa et al. (2012), where the attached vorticity layers were tracked into a resulting vortex pair once the wing vanished from the flow. 
(a)

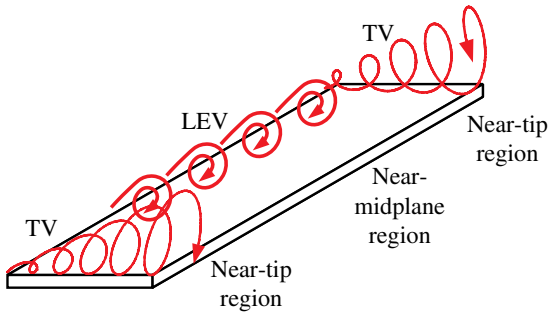

(b)

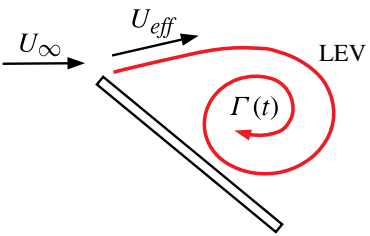

FIGURE 1. (Colour online) Sketch of separated flow over a finite-aspect-ratio plate, including (a) the classification of the two-dimensional and three-dimensional regions according to Jardin et al. (2012) and indicating (b) free stream velocity $U_{\infty}$ and effective shear-layer velocity $U_{\text {eff }}$ as the two characteristic velocities for vortex formation and corresponding circulation $\Gamma$.

Recently, Gazzola, Rees \& Koumoutsakos (2012) studied larval fish during bursts of acceleration, in which entrapped (accelerated) fluid as well as the starting boundary layers were redistributed into the wake. As such, both the initial acceleration of the boundary layer and the subsequent generation of vorticity after the onset of motion seem to play a role in the vortex formation process. In another recent study, Weymouth \& Triantafyllou (2012) showed that the flow retains little memory of its initial conditions upon rapid changes in the fluid-solid interface. It was demonstrated that the vortex shedding from a translating, rapidly shrinking cylinder with radius change from $R_{1}$ to $R_{2}<R_{1}$ (through forced deflation) appears to be identical to the shedding behaviour of a cylinder of constant radius $R_{2}$. It was thus concluded that the initial boundary-layer vorticity was immediately annihilated with opposite-signed vorticity generated during the collapse stage.

As is shown in the above studies, the need for a more rigorous analysis of the vorticity redistribution and reorientation is required, particularly when considering the interaction between leading-edge vortex (LEV) and tip vortex (TV) development on a moving plate. Despite the critical importance of these vortical structures on instantaneous loadings, our ability to control their growth and separation remains elusive. Studies by Ringuette, Milano \& Gharib (2007) and Kim \& Gharib (2010, 2011) have considered the LEV and TV development on low-aspect-ratio flat plates towed from rest in the plate-normal direction. Here it was found that the tip vortex increased the instantaneous drag and that this relative contribution increased with lower aspect ratios. In addition, it was observed that the LEV circulation decreased at spanwise positions closer to the tip. Accordingly, Jardin, Farcy \& David (2012) showed that two major regions exist for accelerating, finite-aspect-ratio plates: a near-midplane region with nominally two-dimensional flow; and a near-tip region with strongly three-dimensional reorientation of vorticity (see figure 1a). Furthermore, they demonstrated that this distinction of the flow topology between two-dimensional and three-dimensional is largely dominated by the chord length rather than by the aspect ratio of the plate. In their study the near-tip region covered approximately one chord inboard from the plate's tip for all considered aspect ratios AR $=2-6$.

Recently Yilmaz \& Rockwell (2012) presented phase-averaged, three-dimensional flow structures showing the LEV and TV growth on low-aspect-ratio rectangular and elliptical flat plates undergoing a pitching motion. In their study, LEV eruption was observed, which was less pronounced for the elliptical planform. Similarly Hartloper, Kinzel \& Rival (2012) investigated the strong time-dependent three-dimensional 
interaction between these above-mentioned vortical structures and showed that for slower accelerations the TV growth was responsible for compressing the LEV in the inboard direction.

In the study of Baik et al. (2012) it is found that, with sufficient forcing (large Strouhal numbers) and large effective angles of attack, vortex growth scales primarily with reduced frequency and that qualitatively the feeding behaviour from the shear layer is the same for all cases. For this reason the current study focuses on a particular accelerating case deemed to be characteristic of such vortical flows found in swimming and flying. However, it should be noted that, for quasi-steady stall or flutter, which lie at extreme ends of the parameter space studied by Baik et al. (2012), the vortex formation processes will differ dramatically from what is presented here.

The objective of the present work is two-fold. First, the growth of vorticity with varying initial conditions is considered by studying the circulation histories in the nominally two-dimensional region of the accelerating plates. Here, the relative influence of the feeding shear layer at the leading edge is studied with regards to LEV development. Also, the persistence of memory from the initial conditions will be examined after the acceleration is completed. The second objective studied here is the three-dimensional reorientation of vorticity near the plate tip, again on the basis of initial conditions and plate kinematics. As such, the relative importance of initial conditions and TV orientation relative to the plate on the long-term force evolution will be considered.

To study the influence of initial conditions at two distinct yet equivalent plate kinematics, two cases have been selected: (i) acceleration of a plate from rest $(U(t=0)=0)$ at a constant angle of attack $\alpha$ to a final towing speed $\dot{h}$; and (ii) perturbation of a developed boundary-layer flow on a flat plate with a sudden cross-stream plunging motion $\dot{h}$ superimposed onto a constant free stream velocity $U_{\infty}$. These two cases will henceforth be referred to as towing and plunging, respectively. In order to contrast these two cases, the two main relevant characteristic velocities - free stream velocity $U_{\infty}$ and effective shear-layer velocity $U_{\text {eff }}$ - must be considered in the development of vortex circulation $\Gamma$, as sketched in figure $1(b)$. As a first approximation, the magnitude and direction of both velocities are effectively identical $\left(U_{\infty}=U_{\text {eff }}\right)$ for pitching and towing motions (see e.g. Koumoutsakos \& Shiels 1996; Yilmaz, Ol \& Rockwell 2010; Buchholz, Green \& Smits 2011; Hartloper et al. 2012). Note that a similar alignment of velocities can be assumed for other unidirectional cases like one-dimensional gusts, as for instance discussed by Afgan et al. (2013). A careful distinction between the two velocities might at first glance seem superfluous if only unidirectional changes in velocity occur.

In contrast, however, these characteristic velocities differ significantly in magnitude and direction $\left(U_{\infty} \neq U_{\text {eff }}\right)$ for plunging motions in cases where the Strouhal number is large, $S t>1$, since the effective shear-layer velocity $U_{\text {eff }}$ is defined to be aligned with the shear-layer orientation, as already indicated by Roshko (1954). Therefore, the orientation and magnitude of $U_{\text {eff }}$ are determined by vector addition of free stream velocity and plate kinematics, i.e. $\boldsymbol{U}_{\text {eff }}=\boldsymbol{U}_{\infty}+\dot{\boldsymbol{h}}$.

When examining these characteristic velocities, on the one hand, it is known that the feeding shear layer controls the growth and thus strength of LEVs through the flux vorticity-containing mass into the LEV, as recently shown by Sattari et al. (2012). Therefore, the advection of circulation into the LEV is a function of the shear layer, with $U_{\text {eff }}$ as the only characterizing velocity measure. This suggests that one should define the kinematics of tow and plunge with identical effective shear-layer velocities $U_{\text {eff }}$ for proper comparison. On the other hand, one could argue that the characteristic 
(a)

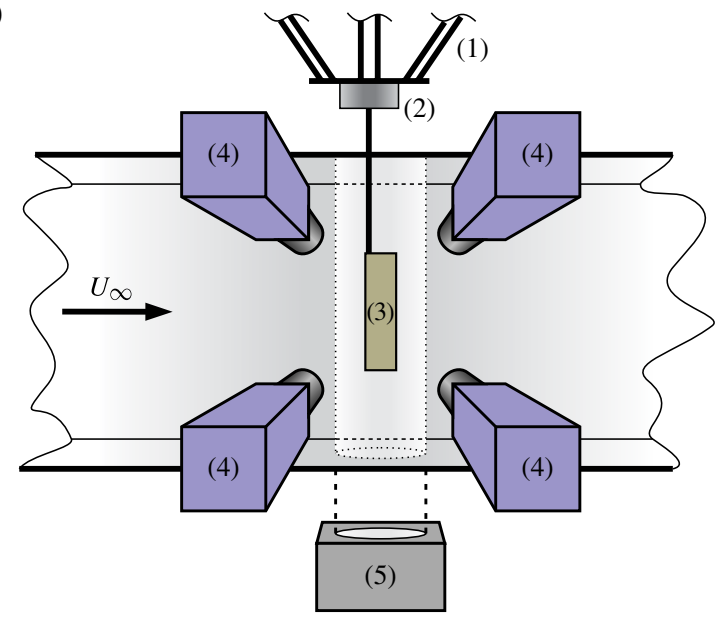

(1) Hexapod manipulator

(2) Six-component force/moment sensor

(3) $\mathrm{AR}=4$ flat plate

(4) pco.edge sCMOS camera system for 3D-PTV

(5) HID light source and lens system

(b)

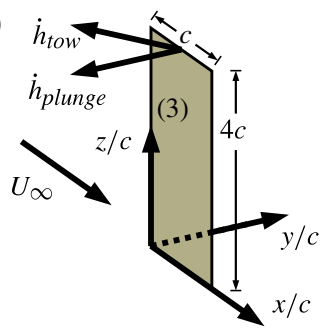

FIgURE 2. (Colour online) (a) Experimental set-up in the water tunnel test section with measurement systems; and $(b) \mathrm{AR}=4$ plate with coordinate system, free stream velocity $U_{\infty}$ and plunge/tow velocities $\dot{h}$.

velocity to compare towed and plunging plates is the free stream velocity $U_{\infty}$ to ensure an identical bulk Reynolds number $R e=c U_{\infty} / v$ for all cases.

Consequently, both characteristic velocities will be considered in the present study in order to clarify this issue. Subsequently, the influence of initial conditions on vortex growth will then be studied for both near-midplane and near-tip regions.

\section{Experimental methods}

All experiments were performed in a free-surface water tunnel at the University of Calgary where a variety of plunging and towing motions for an $A R=4$ plate were controlled by means of a custom hexapod manipulator (see figure 2). The threedimensional particle-tracking velocimetry (3D-PTV) technique, as described by Lüthi, Tsinober \& Kinzelbach (2005), was used to quantify the flow field in the proximity of the flat plates. In order to obtain particle tracks, the water tunnel was seeded with neutrally buoyant $100 \mu \mathrm{m}$ silver-coated, hollow glass spheres with a Stokes number of $S t k \approx 1.2 \times 10^{-3}$. The particles were illuminated by a high-intensity discharge (HID) light source. A lens system (40 and $300 \mathrm{~mm}$ converging lenses) collimated the light to provide a measurement volume with a diameter of $100 \mathrm{~mm}$. The plates were painted black to prevent light scattering, and, together with the orientation of the light column from below, no digital masking was necessary at the image processing stage. During the experiments, raw images were recorded with four pco.edge sCMOS cameras (chip size $2560 \times 2160$ pixels, maximal resolution of $2560 \times 1280$ pixels at 165 frames per second). Simultaneous with the 3D-PTV measurements, direct force measurements were recorded by means of an ATI Gamma six-component force/moment sensor, which was located between the base of the hexapod and the sting holding the plate.

The plate had a chord length of $c=50 \mathrm{~mm}$, a span of $4 c=200 \mathrm{~mm}$ and a thickness of $t / c=6 \%$. Based on the chord length and free stream velocity $U_{\infty}$ or the final towing speed $\dot{h}=0.1 \mathrm{~m} \mathrm{~s}^{-1}$ (depending on the case), the Reynolds number was set to $R e=5000$. Note that the coordinates' point of origin is placed at the intersection of the leading edge and the tip edge as a continuation of the insights reported by 
(a)

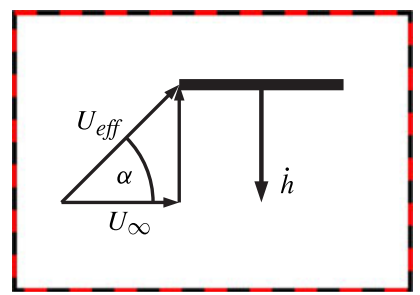

(b)

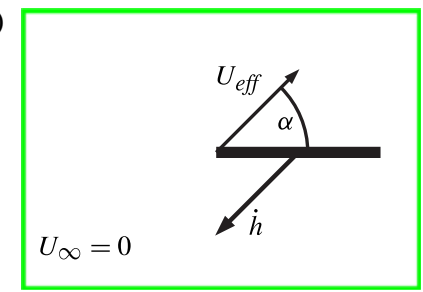

FIGURE 3. (Colour online) The two plate kinematics used in this study. (a) Plunging case(s): plunge to $\alpha_{\text {eff }}=45^{\circ}$ with $U_{\text {eff }}=0.1 \mathrm{~m} \mathrm{~s}^{-1}$ (P45_0.1, red/dark grey) and $U_{\text {eff }}=0.07 \mathrm{~m} \mathrm{~s}^{-1}$ (P45_0.07, black). (b) Towing case: tow to $\dot{h}=0.1 \mathrm{~m} \mathrm{~s}^{-1}$ with $\alpha_{\text {eff }}=45^{\circ}$ (T45_0.1, green/light grey).

Jardin et al. (2012) (see figure 2b). To identify the influence of initial conditions on vortex formation and orientation, as well as the circulation and overall force history, different plate kinematics were applied to the $\mathrm{AR}=4$ flat plate as sketched in figure 3. Superimposed onto a free stream velocity $U_{\infty}=0.1 \mathrm{~m} \mathrm{~s}^{-1}$, the first case $(a)$ corresponds to a plunge velocity $\dot{h}$ of the plate rapidly increasing to $\dot{h}_{\text {final }}=U_{\infty}$. This produces an effective plate angle of $\alpha_{\text {eff }}=45^{\circ}$ in one convective time $t_{\text {eff }}^{*}=t U_{\text {eff }} / c=1$ according to a half-cosine curve (see figure $3 a$ ). As such, a flat-plate boundary layer with corresponding boundary-layer vorticity is the initial condition for this first test case. Note that the insensitivity of the chosen ramp-up motion on the resulting lift forces has been shown by Jones \& Babinsky (2010) and Ol et al. (2010) for rotating and translating wings, respectively.

To investigate the influence of this initial boundary layer on subsequent vortex development, a second case $(b)$ without such a boundary layer is considered, in which the plate is towed from rest at a constant angle of $\alpha_{\text {eff }}=45^{\circ}$ (see figure $3 b$ ). Here the same ramp-up speed as for the first case is applied, such that the towing speed of the plate is increased from rest in one convective time to $\dot{h}_{\text {final }}=U_{\infty}$. The towing case $(b)$ has identical magnitudes for the effective shear-layer velocity $U_{\text {eff }}$ and towing speed $\dot{h}$, whereas in the plunging case $(a)$ the trigonometric relations of $U_{\infty}, \dot{h}$ and $\alpha_{\text {eff }}$ lead to $U_{\text {eff }}=1.41 \dot{h}$. To compensate for this discrepancy, a third case - a modification of case $(a)$ - is tested with appropriately lower velocities, i.e. $U_{\infty}=\dot{h}=0.07 \mathrm{~m} \mathrm{~s}^{-1}$, thus assuring a Reynolds number of $R e_{\text {eff }}=5000$ based on the effective shear-layer velocity $U_{\text {eff }}=0.1 \mathrm{~m} \mathrm{~s}^{-1}$. According to their final $\alpha_{\text {eff }}$ and $\dot{h} / U_{\text {eff }}$ values, the three test cases will be referred to as P45_0.1, P45_0.07 and T45_0.1, where P and T represent plunge and tow, respectively. The resulting curves for $\alpha_{\text {eff }}\left(t^{*}\right)$ and $U_{\text {eff }}\left(t^{*}\right)$, as well as the associated plate accelerations $\ddot{h}\left(t^{*}\right)$, are shown in figure 4 , where the distinction between initial conditions $\left(t^{*}=0\right)$, ramp-up conditions $\left(0 \leqslant t^{*} \leqslant 1\right)$ and constant final conditions $\left(1 \leqslant t_{\text {eff }}^{*} \leqslant 2\right)$ is illustrated. For all cases, $\dot{h}_{\text {final }}$ is held constant after the ramp-up motion $\dot{h}\left(0 \leqslant t^{*} \leqslant 1\right)$ to maintain constant $U_{\text {eff }}$ and $\alpha_{\text {eff }}$ for $1 \leqslant t_{\text {eff }}^{*} \leqslant 2$.

The determination of particle positions and corresponding trajectory information has been performed based on the methods described by Lüthi et al. (2005). First, the Lagrangian velocities and (total) velocity derivatives, $a_{t}$, of the particles were calculated by differentiation of the particle tracks. Subsequently, local and convective accelerations, $a_{l}$ and $a_{c}$, were estimated through weighted interpolation of the Lagrangian information from neighbouring particles. The considered sphere around each particle had an average diameter of $d_{s}=5 \mathrm{~mm}$. Finally all quantities were mapped onto an Eulerian grid with an inter-grid-point spacing of $d_{g}=d_{s}=5 \mathrm{~mm}$. 
(a)

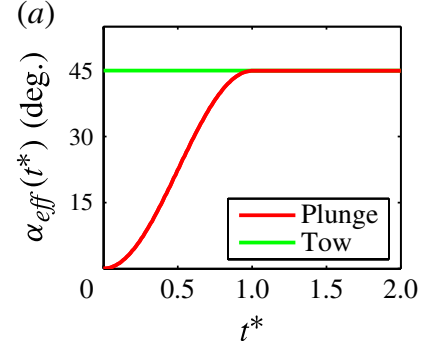

(b)

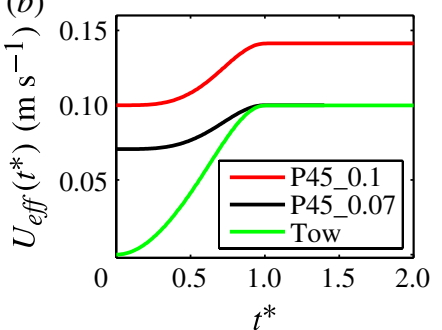

(c)

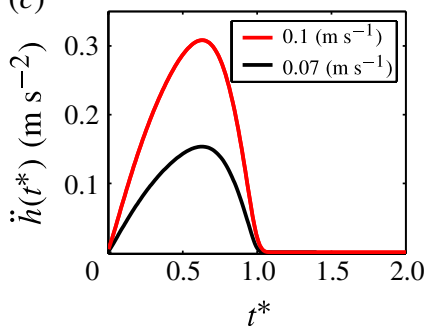

FIgURE 4. (Colour online) Characteristics of the three test cases: (a) angle of attack $\alpha_{\text {eff }}\left(t^{*}\right)$; (b) effective velocity $U_{e f f}\left(t^{*}\right)$; and $(c)$ plate acceleration $\ddot{h}\left(t^{*}\right)$. Initial conditions, $t^{*}=0$; rampup, $0 \leqslant t^{*} \leqslant 1$; constant final conditions, $1 \leqslant t^{*} \leqslant 2$.

The relative uncertainty in the particle-track velocity was estimated to be $\delta u / u<$ \pm 0.01 using the method described by Feng, Goree \& Liu (2011). The accuracy of the velocity-derivative calculations was estimated by comparing the Lagrangian acceleration $a_{t}=\mathrm{D} u_{i} / \mathrm{D} t$ with the sum of the local acceleration $a_{l}=\partial u_{i} / \partial t$ and convective acceleration $a_{c}=u_{j} \partial u_{i} / \partial x_{j}$ (see Lüthi et al. 2005). Calculation of the normalized error resulting from the acceleration $\sigma_{a}$ based on

$$
\sigma_{a}=\frac{1}{3} \sum_{i=x, y, z} \frac{\left|a_{t}^{i}-a_{l}^{i}-a_{c}^{i}\right|}{\left|a_{t}^{i}\right|+\left|a_{l}^{i}\right|+\left|a_{c}^{i}\right|}, \quad \text { with } \sigma_{a} \in[0,1],
$$

resulted in an uncertainty estimate of $\sigma_{a}=0.1$. Figure 5 exhibits some exemplary raw PTV results for the plunging case P45_0.1. From the Eulerian data, iso-surfaces of non-dimensional vorticity $\omega^{*}=\omega c / U_{\infty}=2.5$ were extracted to identify coherent vortical structures, as shown in figure 5(a), which reveal the LEV, TV and some trace of the trailing-edge vortex (TEV). The corresponding particle tracks are shown in figure $5(b)$, where the colour coding (greyscale shading) represents the convective time $t^{*}=t U_{\infty} / c$ elapsed during the plate motion. To better demonstrate the spatial separation of the nominal two-dimensional (near-midplane) and three-dimensional (near-tip) regions, figure 5(c) shows the identical tracks as presented in figure 5(b) but in the YZ-plane. Further analysis of all three vorticity components $\omega_{i}$ is performed based on spatial integration across the chosen $X Y$-planes (cf. figure $5 a$ ) according to

$$
\Gamma^{i}=\iint_{\substack{X Y \\ \text { plane }}} \omega_{i} \mathrm{~d} x \mathrm{~d} y,
$$

where $\Gamma^{i} \hat{=} \Gamma^{x}, \quad \Gamma^{y}$ and $\Gamma^{z}$ are based on streamwise, plate-normal and spanwise vorticity components, respectively. A threshold value of $\omega_{t h}=0.05$ has been applied to (2.2) for all circulation calculations, i.e.

$$
\omega_{i}\left(\omega_{i}<\omega_{t h}\right) \stackrel{!}{=} 0,
$$

which assured the removal of background noise. To avoid confusion during the discussion of the near-midplane results, the variable for spanwise circulation will initially be written without superscript, i.e. $\Gamma^{z} \stackrel{!}{=} \Gamma$ and will be simply referred to as circulation. 
(a)
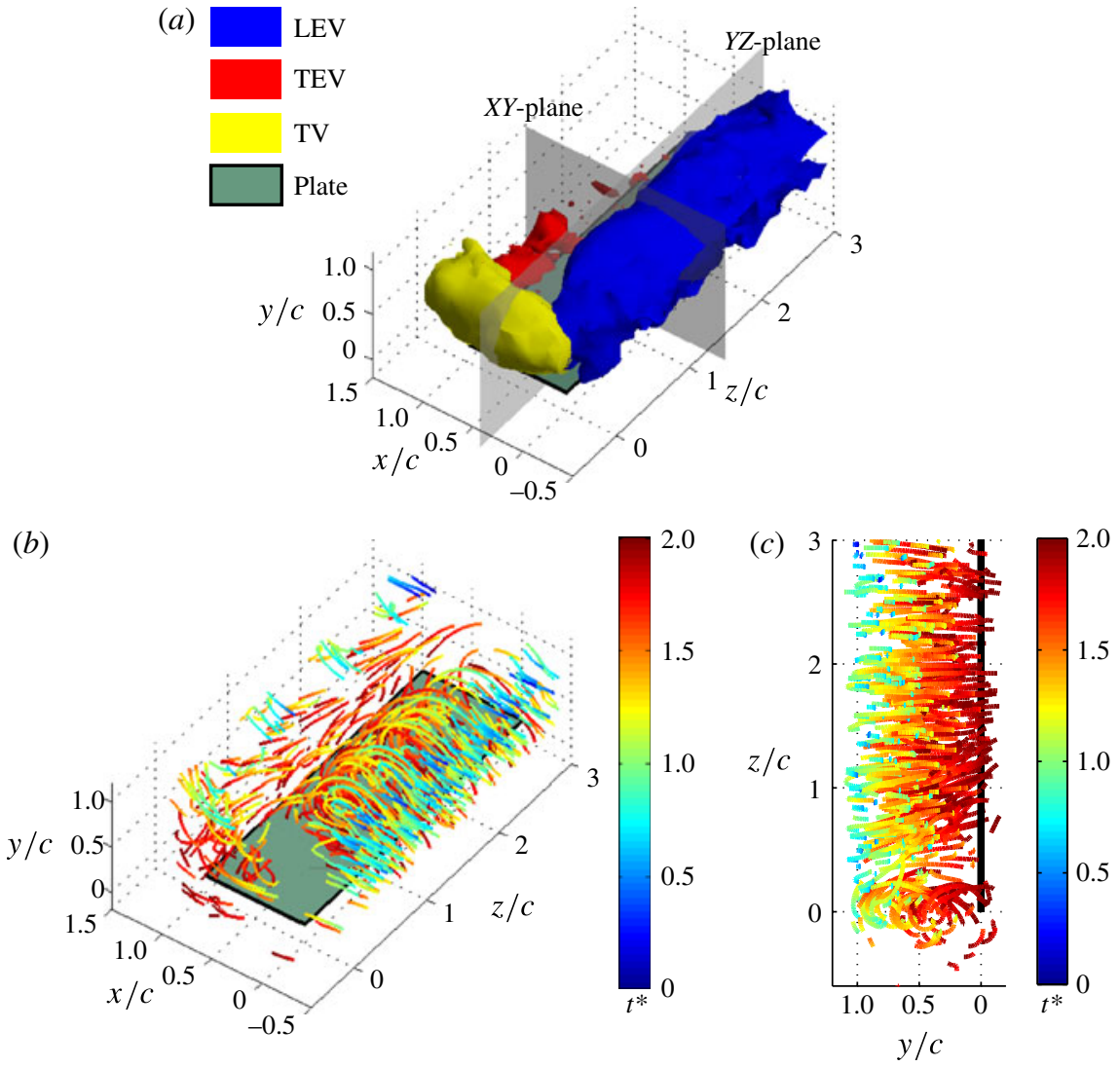

FIGURE 5. (Colour online) Exemplary PTV data (P45_0.1) identifying the two-dimensional (near-midplane) and three-dimensional (near-tip) regions: (a) Eulerian iso-surfaces of vorticity $\left(\omega^{*}=2.5\right)$; $(b)$ Lagrangian particle tracks; and $(c)$ two-dimensional versus threedimensional regions.

\section{Results}

To verify the nominal two-dimensional character of the near-midplane region, circulation plots at different spanwise locations are shown in figure 6 for the plunging case (P45_0.1). Beyond one chord inboard from the tip $(z / c>1)$, the curves of the LEV circulation collapse within the measurement accuracy. Therefore, in the current study, the spanwise location $1 \leqslant z / c \leqslant 3$ is assumed as the near-midplane region with bulk two-dimensional character. Following this distinction, the discussion of the experimental results starts with the near-midplane region in $\S 3.1$. Subsequently, the two-dimensional results are compared with the total force-sensor data in $\S 3.2$. The comparison between the force measurements and the flow in the near-midplane region for the different experimental configurations will show the importance of the near-tip region $(z / c<1)$ to the overall force production. Finally, a discussion on the flow in this region is presented in $\S 3.3$.

\subsection{Near-midplane region}

In order to characterize the influence of varying initial conditions on the subsequent vortex formation, the LEV circulation histories in the near-midplane region 


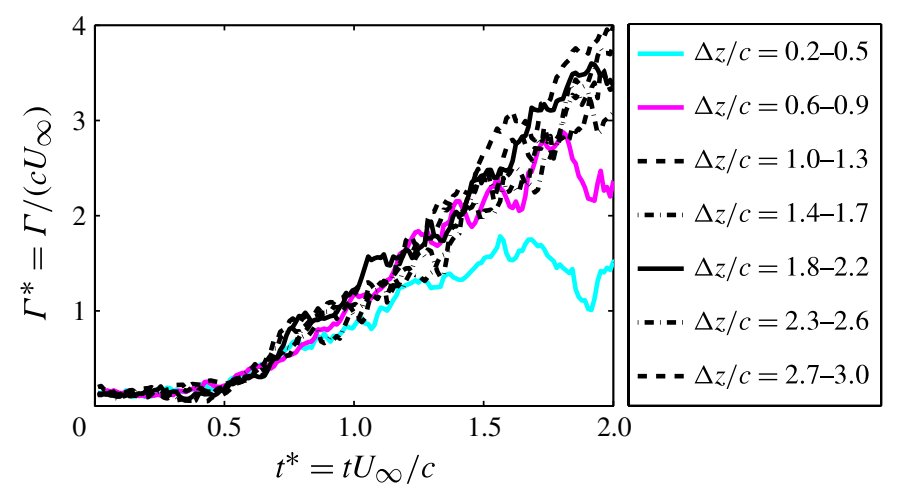

FIGURE 6. (Colour online) LEV circulation for different spanwise locations $z / c$ of the plunging case (P45_0.1).
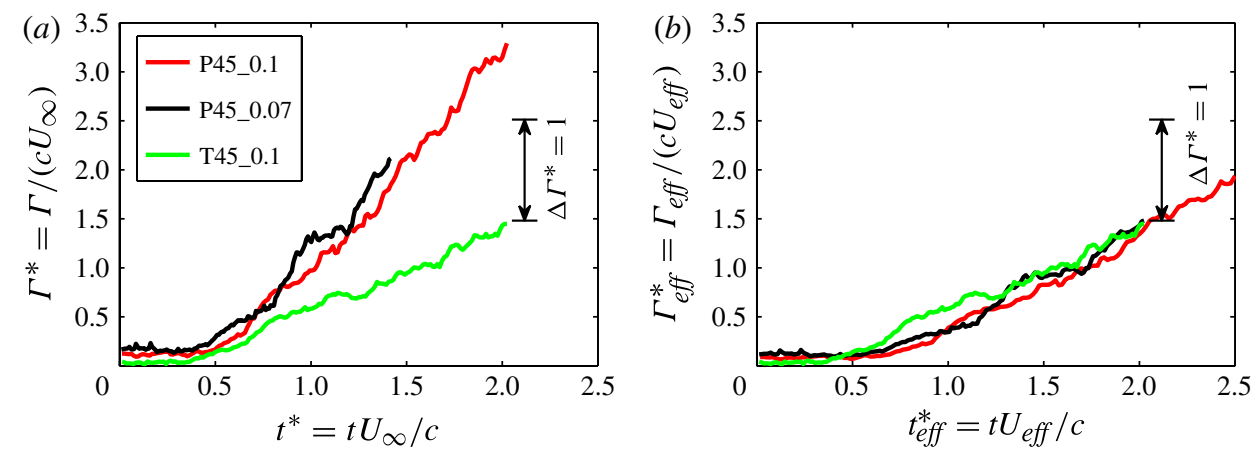

FIGURE 7. (Colour online) LEV circulation in the near-midplane region $(1 \leqslant z / c \leqslant 3)$ for all four test cases: $(a) \Gamma^{*}$ and $t^{*}$ normalized with free stream velocity $U_{\infty} ;(b) \Gamma_{e f f}^{*}$ and $t_{e f f}^{*}$ normalized with the effective velocity $U_{\text {eff }}$ instead.

$(1 \leqslant z / c \leqslant 3)$ for all three test cases are compared in figure 7. In figure 7(a) the nondimensional circulation $\Gamma^{*}=\Gamma /\left(c U_{\infty}\right)$ and convective time $t^{*}=t U_{\infty} / c$ are normalized with the free stream velocity $U_{\infty}$, which corresponds to the final tow speed $\dot{h}$ for the towing case. As discussed in the introduction, owing to the additional boundary-layer vorticity $\Gamma_{B L}^{*}=\Gamma_{B L} /\left(c U_{\infty}\right)=1$, one would expect a constant offset of $\Delta \Gamma^{*}=\Gamma_{B L}^{*}=1$ for the circulation histories between the plunging case P45_0.1 and the towing case T45_0.1, as sketched in figure 7(a). However, the difference between both plunging cases and T45_0.1 shows an offset of $\Delta \Gamma^{*} \approx 2$. In order to explore the cause of this larger offset, the free stream velocity $U_{\infty}$ is replaced with the effective shear-layer velocity $U_{\text {eff }}$ to normalize circulation and time, i.e. $\Gamma_{\text {eff }}^{*}=\Gamma /\left(c U_{\text {eff }}\right)$ and $t_{\text {eff }}^{*}=t U_{\text {eff }} / c$ (cf. $U_{s}$ in figure $1 b$ ). This is particularly sensible since the vortex growth rate is determined by both the generation of vorticity and advection of circulation at the leading edge, each of which is limited by $U_{\text {eff }}$.

The non-dimensionalization used in figure $7(b)$ reveals a collapse for the two plunging cases together with the towing case T45_0.1. Despite the varying initial conditions between plunging and towing, it must be concluded that the initial boundary-layer vorticity, at least in the near-midplane region, plays little role in 
the vortex formation process. In other words, the initial layer of vorticity must be rapidly annihilated with an opposite-signed layer on the plate surface once the motion begins. Recall that Weymouth \& Triantafyllou (2012) report a similar disappearance of surface-layer vorticity for a rapidly shrinking cylinder. Therefore, it has to be concluded that rapid acceleration of fluid boundaries (e.g. moving or morphing obstacles) accelerates fluid with opposite-signed vorticity towards an existing boundary layer, which leads to the sudden cross-annihilation of the two vorticity layers.

Both findings - collapse and loss of memory - are confirmed by LEV formation plots: figure 8 shows the temporal evolution and corresponding spatial distribution of spanwise vorticity $\omega_{z, \text { eff }}^{*}$ for the near-midplane region ( $X Y$-plane) of all three cases. The $\omega_{z, \text { eff }}^{*}$ iso-lines reveal a similar growth of the LEV for all three cases, which reaffirms the insights obtained from the circulation plots of the near-midplane region (see figure 7). However, after the acceleration of the plate stops at $t^{*} \geqslant 1$, it can be observed that the tow case LEV starts to roll off the plate. Despite identical circulation, as demonstrated in figure 7, this beginning roll-off might adversely affect the resulting magnitude of the lift force for the towing case.

It is important to note that the plate-fixed frame of reference in fact is an accelerated reference frame. As such, the (attached) vortical structures are accelerated according to the orientation of the respective plate kinematics (i.e. acceleration $\ddot{\boldsymbol{h}}$ ) in an inertial system. It is therefore hypothesized that the remaining inertia of the accelerated fluid causes the roll-off of the tow case LEV once the plate acceleration stops.

\subsection{Direct force measurements}

It has been demonstrated that the circulation history $\Gamma_{L E V}^{*}$ in the near-midplane region of the $\mathrm{AR}=4$ plates is identical for both plunging cases and T45_0.1. The corresponding total force results are shown in figure 9. Here, lift $C_{l}$ and drag $C_{d}$ forces are defined to be perpendicular and parallel to the direction of the plate velocity, respectively (upper row). In addition, $C_{y}$ and $C_{x}$ representing the plate-normal and plate-parallel forces are plotted for comparison, respectively (lower row). All force data are non-dimensionalized based on the standard convention $\left(U_{\infty}\right)$ on the left but also using $U_{\text {eff }}$ on the right.

First, it is worth noting that the curves for the plunging motions show unexpectedly high values when normalized with $U_{\infty}$. In particular, for $1 \leqslant t^{*} \leqslant 2$ and $t_{\text {eff }}^{*}<1$, the force magnitudes for T45_0.1 and P45_0.07 should be similar since the LEV is fed with the same shear-layer velocity at identical effective angles of attack. This discrepancy confirms the validity of $U_{\text {eff }}$ as the characterizing velocity scale to describe vortex growth and thus resulting forces.

Furthermore, it is remarkable that the force histories of both plunging cases (P45_0.1 and P45_0.07) share identical curves during the plate acceleration for $t^{*}<1$ and $t_{\text {eff }}^{*}<1$ despite the fact that they experience different magnitudes of acceleration $\ddot{h}$ during the respective plate motions (see figure $4 c$ ). In contrast, the slow plunge and the tow case (P45_0.07 and T45_0.1) obviously yield significantly different force histories, even though in both cases the magnitude of plate acceleration was identical. Consequently, it is concluded that the differences in the force histories of the three contrasted cases are not due to acceleration effects.

However, the direct comparison of the rapidly increasing lift (figure $9 b$ ) to values $C_{l} \geqslant 2$ for $t_{e f f}^{*}<1$, with the final values of $\Gamma_{L E V}^{*}\left(t_{\text {eff }}^{*}=2\right) \approx 1.5$ shown in figure $7(b)$, emphasizes the important role of the tip vortex as the only remaining contributor on the overall force production (see e.g. Ringuette et al. 2007). 

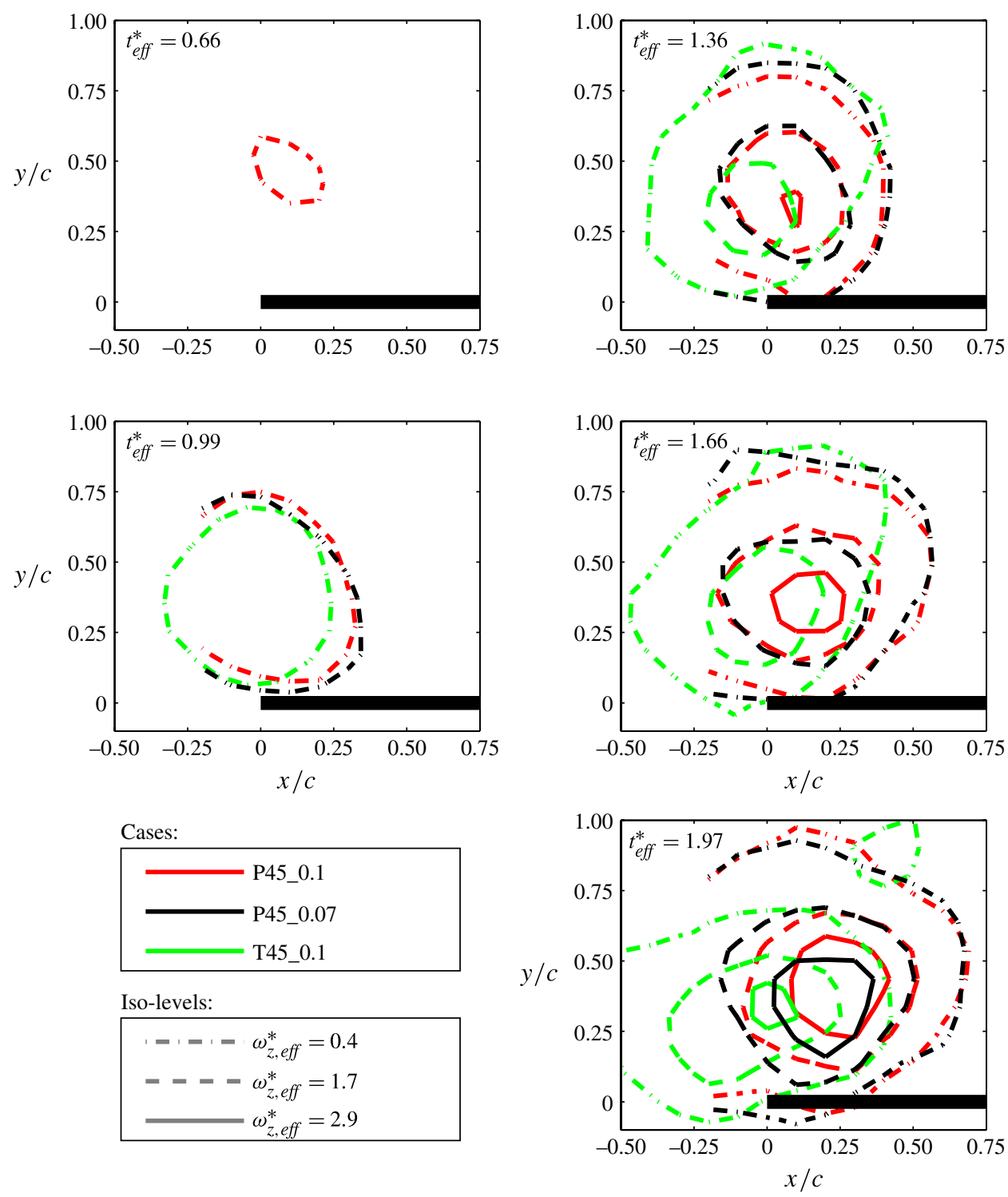

FIGURE 8. (Colour online) LEV formation in the near-midplane region: spatial distribution of spanwise vorticity $\omega_{z, \text { eff }}^{*}$ for five different time steps $t_{\text {eff }}^{*}=0.66,0.99,1.36,1.66,1.97$. Left column: ramp-up motion $\left(0 \leqslant t_{\text {eff }}^{*} \leqslant 1\right)$. Right column: constant final conditions $\left(1 \leqslant t_{\text {eff }}^{*} \leqslant 2\right)$.

Figure $9(d)$ reveals a sharp drop of plate-normal force $C_{y}=\left(2 f_{y}\right) /\left(\rho A U_{\text {eff }}^{2}\right)$ for the towing case at $t_{\text {eff }}^{*}=1$. Recall that the above-mentioned LEV roll-off occurs gradually for $t_{\text {eff }}^{*}>1$ (see figure 8). Therefore, it is concluded that the observed $C_{y}$ drop does not occur due to the relative LEV motion. Consequently, the discrepancy between the $C_{y}$ slopes for plunge and tow cases in figure $9(d)$ suggest that, in addition to the presence of a tip vortex, its orientation relative to the plate surface might influence the resulting force production. Both of these results require further interpretation of the vorticity 


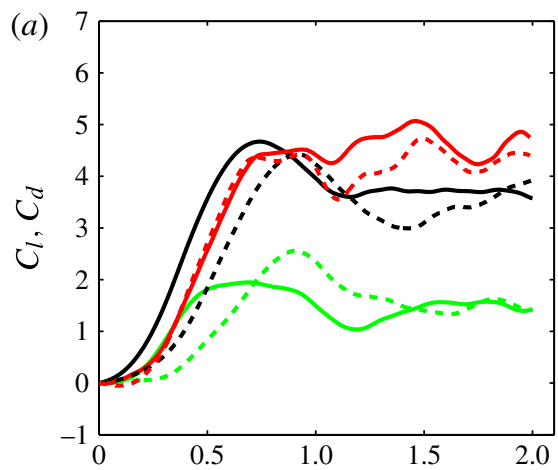

(b)
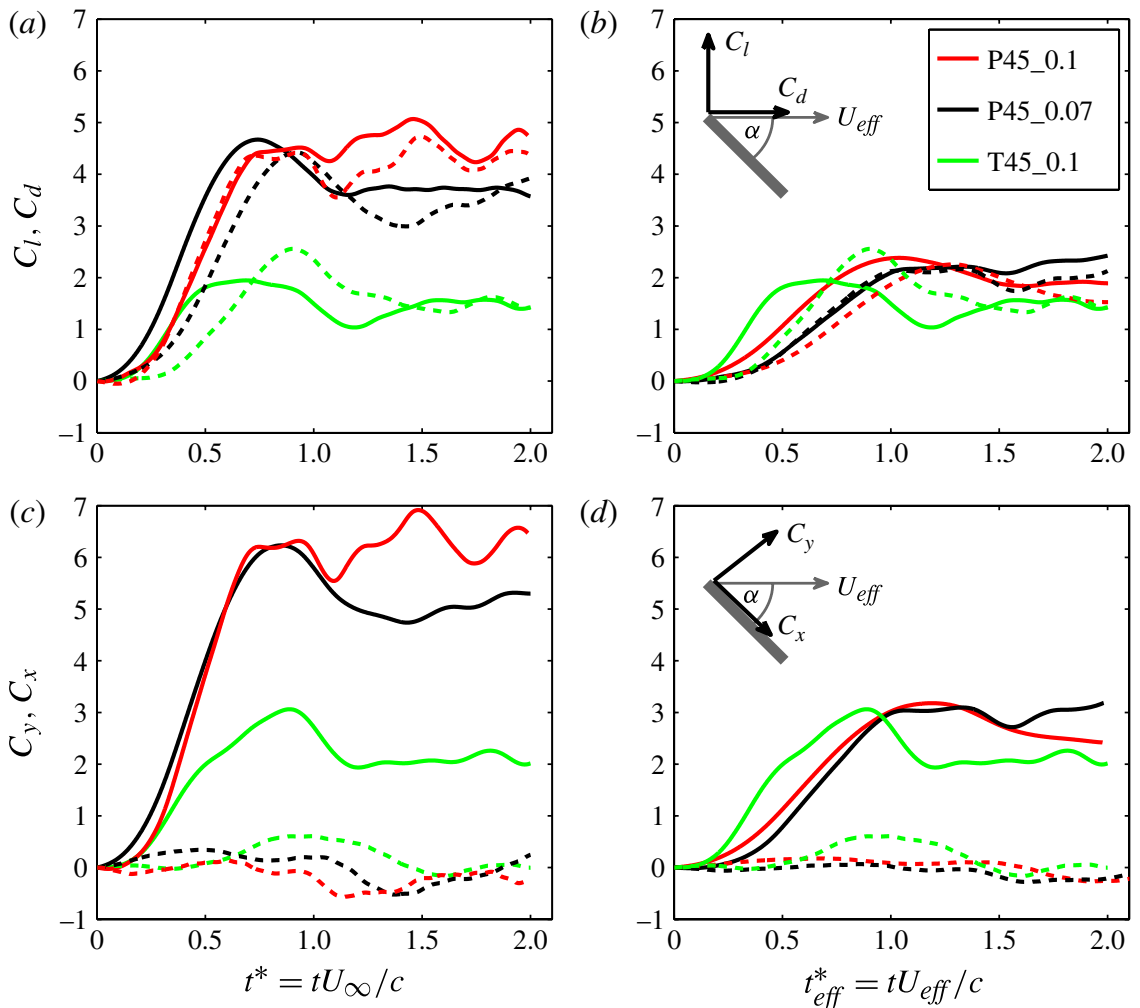

(d)

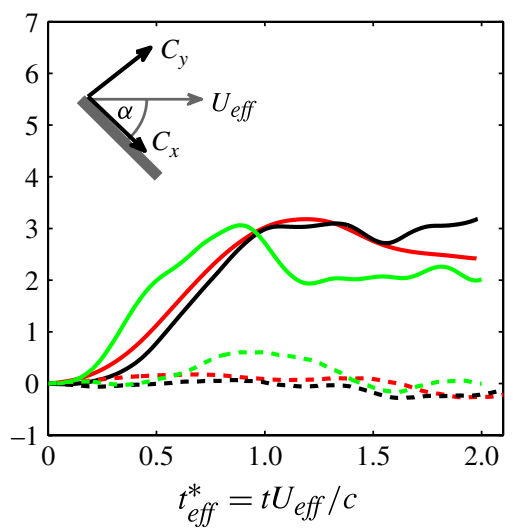

FIGURE 9. (Colour online) Force data for all four cases: $(a, b)$ the lift force $C_{l}(-)$ and drag force $C_{d}(---)$ normalized with $(a) U_{\infty}$ and $(b) U_{\text {eff }} ;(c, d)$ the plate-normal force $C_{y}(-)$ and plate-parallel force $C_{x}(---)$ normalized with $(c) U_{\infty}$ and $(d) U_{\text {eff }}$.

redistribution and reorientation in the near-tip region of the $\mathrm{AR}=4$ plate, as will be performed for P45_0.1 and T45_0.1 in the following section.

\subsection{Near-tip region}

As mentioned earlier, Ringuette et al. (2007) emphasized the important role of tip vortices on the resulting unsteady loadings of finite-aspect-ratio plates. Furthermore, Buchholz et al. (2011) recently addressed the influence of aspect ratio (and stroke amplitude) on the circulation of such pitching plates. In their work they proposed a correction factor for non-dimensional circulation, which considers span and pitch amplitude of the plate so as to correct the determined plate circulation for varying AR.

To confirm both reports' findings, the circulation histories $\Gamma_{e f f}^{i, *}$ are calculated according to (2.2) as a function of span $z / c$ and time $t_{\text {eff }}^{*}$. The resulting spatio-temporal evolution of all three vorticity components is provided in figure 10. In accordance with the reports of Jardin et al. (2012), neither streamwise (tip) nor plate-normal vorticity are significant in the midplane region, which supports the previous assumption of two-dimensional LEV formation.

The comparison of figures $10(a)$ and $10(c)$ indicates that both LEV and TV strengths increase simultaneously. The LEV-TV competition, as described by Hartloper et al. (2012), leads to a deformation of the LEV, resulting in the production of plate-normal vorticity as shown in figure $10(b)$. Recall that, despite identical 
(a)

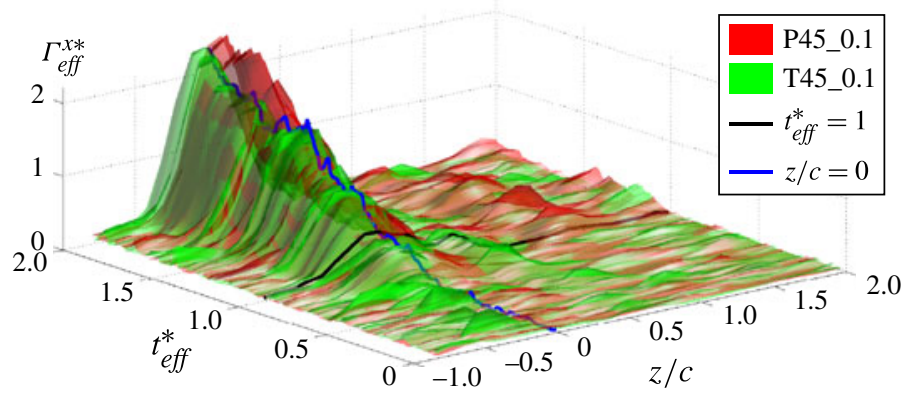

(b)

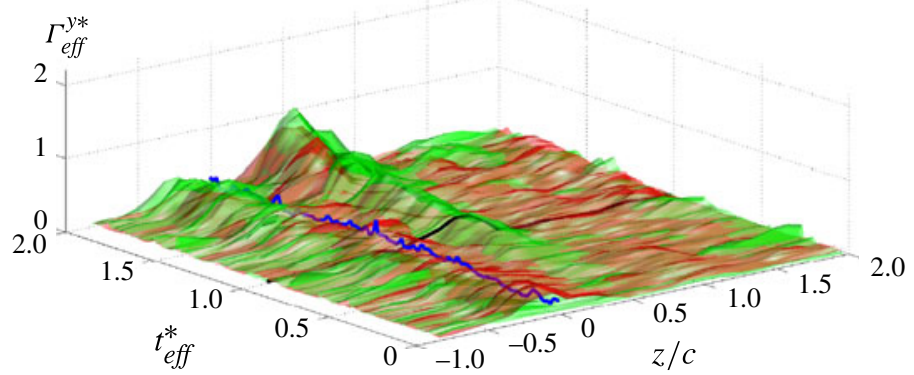

(c)

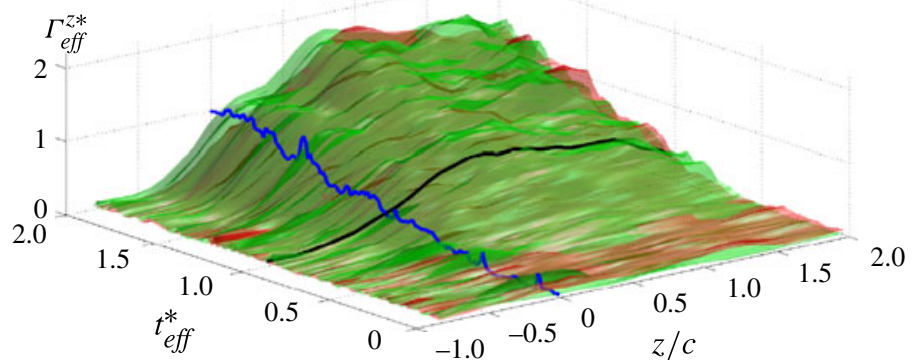

FIGURE 10. (Colour online) Spatio-temporal vorticity distribution as function of span $z / c$ and time $t_{\text {eff }}^{*}=t U_{\text {eff }} / c$ for plunging (red/dark grey) and towing (green/light grey) cases: (a) streamwise vorticity $\omega_{x}$; (b) plate-normal vorticity $\omega_{y}$; and $(c)$ spanwise vorticity $\omega_{z}$. The blue (dark grey) and black lines are included to show $z / c=0$ (tip location) and $t^{*}=1$ (end of acceleration) for the sake of clarity.

spanwise distributions and temporal evolutions of all vorticity components for the two kinematics shown, the plate-normal force coefficient $C_{y}$ is significantly higher in the plunging case when compared to the towing case (see figure $9 d$ ). Consequently, further analysis of the vortical structures beyond only integral values is required to characterize the difference between the two contrasted cases.

Lagrangian tracks of the near-tip region for the plunging case P45_0.1 and towing case T45_0.1 are shown in figure 11. Even though both diagrams clearly indicate the presence of the TV, the trajectories differ between the two. This suggests that the varying TV orientations relative to the plate surface might play a critical role in force generation and thus requires a further quantitative analysis of the vorticity distribution.

Analogous to LEV development as shown in figure 8, the corresponding TV formation is exhibited in figure 12 in a $Y Z$-plane at $x / c=0.6$, where instead the 

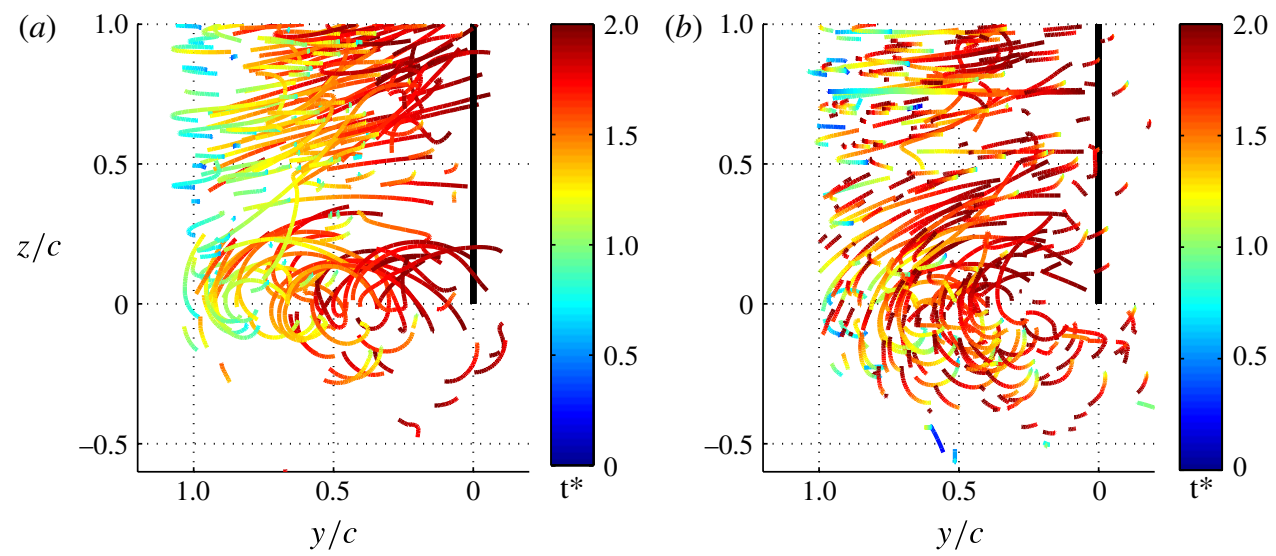

FIGURE 11. (Colour online) Lagrangian particle tracks in the near-tip region $z / c<1$ (cf. figure 5): (a) plunge P45_0.1; and (b) tow T45_0.1.

streamwise component of vorticity is plotted. The iso-lines of streamwise vorticity $\omega_{x, \text { eff }}^{*}$ demonstrate that the TV forms simultaneously to the LEV, which confirms the findings from the spatio-temporal analysis (see figure 10). The slow plunge case P45_0.07 is added to the diagrams to confirm the similarity of both plunging cases' results.

Interestingly, an almost instantaneous lift-off of the plate can be identified for the tow case after the plate acceleration stops at $t_{\text {eff }}^{*}>1$. This constant offset of $\omega_{x, \text { eff }}^{*}$ isolines already suggests that the tow case TV contributes less effectively to lift. To study the shape and orientation of the entire vortical structure more rigorously, iso-surfaces of streamwise vorticity are plotted for both cases in figure 13 relative to the plate surface for time steps before $\left(t_{\text {eff }}^{*}=0.95\right)$ and after $\left(t_{e f f}^{*}=1.43\right)$ the variation in forces is observed. Figure 13(a) demonstrates that prior to the force-data offset $\left(t_{e f f}^{*}<1\right)$ both location and orientation of both TVs are essentially identical (cf. figure 12, left column).

In contrast, figure $13(b)$ demonstrates that the entire TV structure of the towing case shows the above-mentioned offset, whereas the TV for the plunging case remains attached to the surface. Even though the two TVs are still of similar strength, the detached TV in the towing case cannot contribute to lift. This effect is of prime importance since it must be concluded that, in addition to the reorientation of vorticity, the relative positioning of such vortical structures relative to the plate dominate the production of instantaneous lift once the plate acceleration has ceased.

\section{Conclusions}

An investigation into the influence of initial conditions on vortex formation for rapidly accelerating, $\mathrm{AR}=4$ plates has been performed. In addition, the vorticity redistribution and reorientation in the plate's proximity has also been studied. The data were obtained simultaneously by means of a 3D-PTV system together with a six-component force/moment sensor.

In the two-dimensional analysis of the plate's near-midplane region, as defined by Jardin et al. (2012), it has been shown that the effective velocity $U_{\text {eff }}$ of the feeding shear layer is the appropriate characteristic velocity rather than the more 

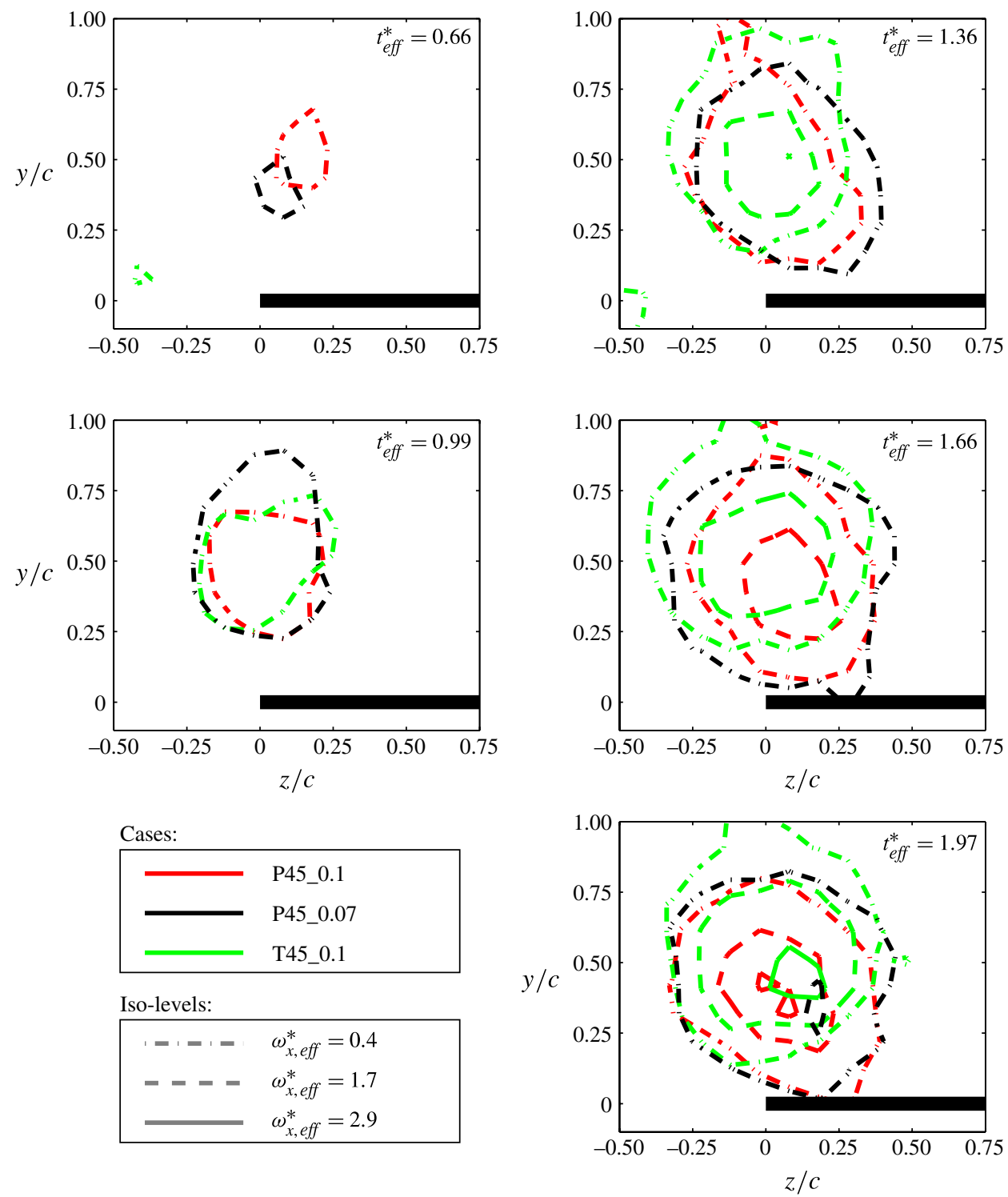

FIGURE 12. (Colour online) TV formation in the near-tip region at $x / c=0.6$ : spatial distribution of streamwise vorticity $\omega_{x, \text { eff }}^{*}$ for five different time steps $t_{\text {eff }}^{*}=$ $0.66,0.99,1.36,1.66,1.97$. Left column: ramp-up motion $\left(0 \leqslant t_{\text {eff }}^{*} \leqslant 1\right)$. Right column: constant final conditions $\left(1 \leqslant t_{\text {eff }}^{*} \leqslant 2\right)$.

commonly used plunge $\dot{h}$ or free stream $U_{\infty}$ velocities. It is therefore proposed that the non-dimensionalization for various plate kinematics should be based on the effective feeding shear-layer velocity since it dictates vortex growth and thus overall force histories. Interestingly, based on $U_{\text {eff }}$ as the characteristic velocity, the curves for differing ramp-up speeds collapsed, suggesting that this feeding velocity is more critical than any Reynolds-number effects. 
(a)

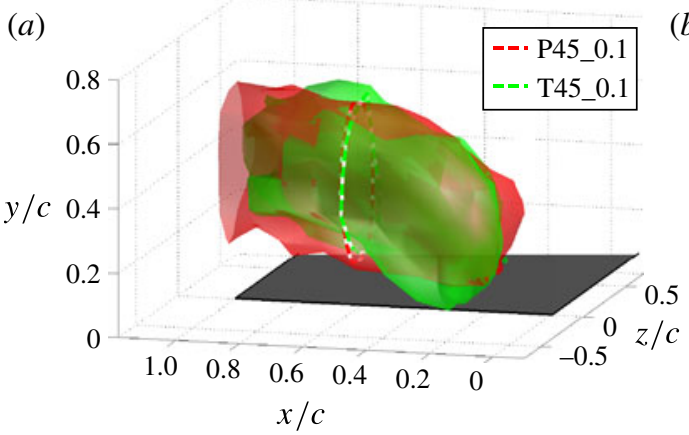

(b)

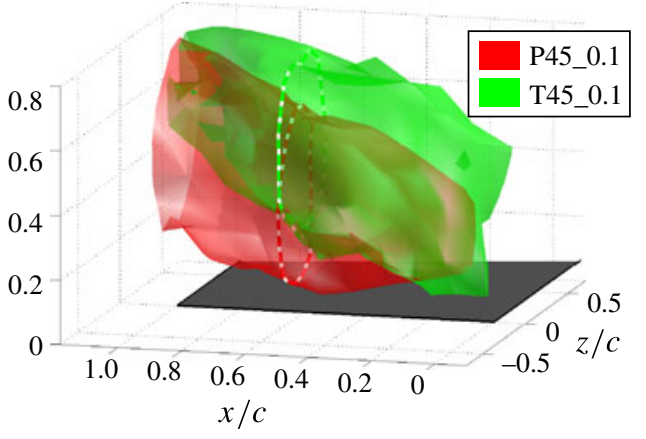

FIGURE 13. (Colour online) Direct comparison of tip-vortex location for plunging and towing cases (iso-surface value $\omega_{x, \text { eff }}^{*}=1.9$; dashed isolines indicate $Y Z$-plane at $x / c=0.6$ ): (a) $t_{\text {eff }}^{*}=0.95 ;$ and $(b) t_{\text {eff }}^{*}=1.43$.

Based on these newly normalized circulation histories, it has then been demonstrated that there is in fact no persistence of memory from the initial conditions. The existence of initial boundary-layer vorticity on the plunging plate - at least in the near-midplane region - does not contribute to the eventual vortex formation process. Similar to the findings from Weymouth \& Triantafyllou (2012), it is concluded that, shortly after the motion begins, the rapid acceleration of fluid with opposite-signed vorticity cross-annihilates the initial layer of vorticity.

In accordance with the reports of Ringuette et al. (2007), TV has been identified to be of critical importance in the overall force production. It is found that the vortex orientation relative to the plate surface influences the instantaneous force once acceleration ceases. In particular, the comparative study of towing and plunging kinematics uncovered differing orientations of the respective TVs. The observed offset occurred despite identical effective angle of attack $\alpha_{\text {eff }}$ and shear-layer velocity $U_{\text {eff }}$. It has been demonstrated that, for the towing case, once the TV detaches from the plate, it contributes little to lift.

\section{Acknowledgements}

The authors gratefully acknowledge funding from the Natural Sciences and Engineering Research Council of Canada. The authors would also like to thank the reviewers for their valuable feedback.

\section{REFERENCES}

Afgan, I., Benhamadouche, S., Han, X., Sagaut, P. \& Laurence, D. 2013 Flow over a flat plate with uniform inlet and incident coherent gusts. J. Fluid Mech. 720, 457-485.

Baik, Y., Bernal, L., Granlund, K. \& OL, M. 2012 Unsteady force generation and vortex dynamics of pitching and plunging aerofoils. J. Fluid Mech. 709, 37-68.

Buchholz, J. H. J., Green, M. A. \& Smits, A. J. 2011 Scaling the circulation shed by a pitching panel. J. Fluid Mech. 688, 591-601.

Feng, Y., Goree, J. \& LiU, B. 2011 Errors in particle tracking velocimetry with high-speed cameras. Rev. Sci. Instrum. 82, 053707.

Gazzola, M., Van Rees, W. M. \& Koumoutsakos, P. 2012 C-start: optimal start of larval fish. J. Fluid Mech. 698, 5-18. 
GENDRICH, C. P. 1999 Dynamic stall of rapidly pitching airfoils: MTV experiments and Navier-Stokes simulations. PhD thesis, Michigan State University.

Hartloper, C., Kinzel, M. \& Rival, D. 2012 On the competition between leading-edge and tip-vortex growth for a pitching plate. Exp. Fluids 54, 1447.

JARDin, T., FARCY, A. \& DAVID, L. 2012 Three-dimensional effects in hovering flapping flight. J. Fluid Mech. 702, 102-125.

Jones, A. R. \& BABINSKY, H. 2010 Unsteady lift generation on rotating wings at low Reynolds numbers. J. Aircraft 47 (3), 1013-1021.

KIM, D. \& Gharib, M. 2010 Experimental study of three-dimensional vortex structures in translating and rotating plates. Exp. Fluids 49, 329-339.

KIM, D. \& GHARIB, M. 2011 Flexibility effects on vortex formation of translating plates. J. Fluid Mech. 677, 255-271.

Koumoutsakos, P. \& Shiels, D. 1996 Simulations of the viscous flow normal to an impulsively started and uniformly accelerated flat plate. J. Fluid Mech. 328, 177-227.

LÜthi, B., Tsinober, A. \& KinZelbaCh, W. 2005 Lagrangian measurement of vorticity dynamics in turbulent flow. J. Fluid Mech. 528, 87-118.

Morton, B. R. 1984 The generation and decay of vorticity. Geophys. Astrophys. Fluid Dyn. 28 (3-4), 277-308.

Ol, M. V., Altman, A., Eldredge, J. D., Garmann, D. J. \& Lian, Y. 2010 Résumé of the AIAA FDTC Low Reynolds Number Discussion Group's canonical cases. In Proceedings of the 48th AIAA Aerospace Sciences Meeting, 4-7 January 2010, Orlando, FL, USA. AIAA 2010-1085.

Ringuette, M. J., Milano, M. \& Gharib, M. 2007 Role of the tip vortex in the force generation of low-aspect-ratio normal flat plates. J. Fluid Mech. 581, 453-468.

Roshko, A. 1954 On the drag and shedding frequency of two-dimensional bluff bodies. NACA Tech. Rep. TN 3169.

Sattari, P., Rival, D. E., Martinuzzi, R. J. \& Tropea, C. 2012 Growth and separation of a start-up vortex from a two-dimensional shear layer. Phys. Fluids 24, 107102.

Weymouth, G. D. \& Triantafyllou, M. S. 2012 Global vorticity shedding for a shrinking cylinder. J. Fluid Mech. 702, 470-487.

White, F. M. 1991 Viscous Fluid Flow, 2nd edn, pp. 137-143. McGraw-Hill.

Wibawa, M. S., Steele, S. C., Dahl, J. M., Rival, D. E., Weymouth, G. D. \& Triantafyllou, M. S. 2012 Global vorticity shedding for a vanishing wing. J. Fluid Mech. 695, 112-134.

Yilmaz, T., Ol, M. \& Rockwell, D. 2010 Scaling of flow separation on a pitching low aspect ratio plate. J. Fluids Struct. 26, 1034-1041.

Yilmaz, T. O. \& RockWell, D. 2012 Flow structure on finite-span wings due to pitch-up motion. J. Fluid Mech. 691, 518-545. 